\title{
Metronidazole neurotoxicity in late liver transplantation
}

Neurotoxidade por metronidazol em pós-operatório de transplante de fígado

Livia Pinheiro de OLIVEIRA', Paula Teixeira MARQUES' ${ }^{1}$, Marcos Cristhiano LANGE'

A 67-year-old female developed subacute peripheral neuropathy, nystagmus and encephalopathy. She was on continuous metronidazole use for 65 days for a liver abscess after receiving a transplantation. Diagnostic workup revealed a sensory axonal polyneuropathy in electroneuromyography. Brain magnetic resonance imaging (MRI) showed hyperintensities at the dentate nucleus and pons (Figure 1). Metronidazole treatment was discontinued and within the first week the patient evolved with complete improvement of encephalopathy. However, she maintained the

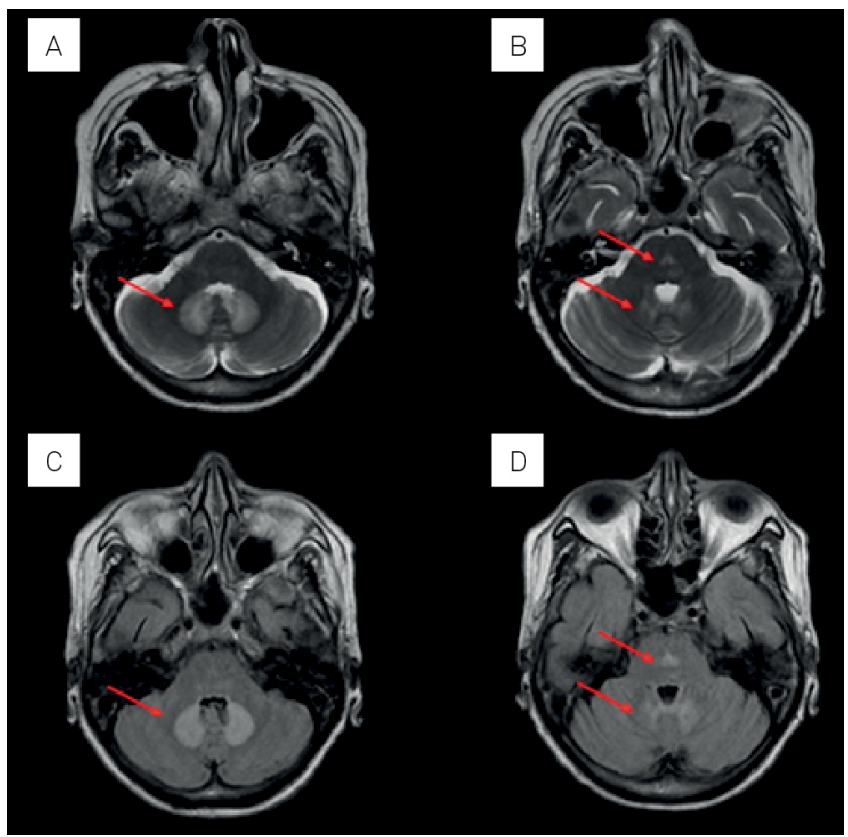

Figure 1. Brain MRI with symmetric hyperintensity on axial T2-weighted images at the dentate nucleus (A, B) and pons (B), and increased signal on axial FLAIR images (C, D). neuropathy symptoms. A control brain MRI was normal after 30 days (Figure 2). Metronidazole neurotoxicity was previously reported with these same imaging findings and clinical course $e^{1-4}$.

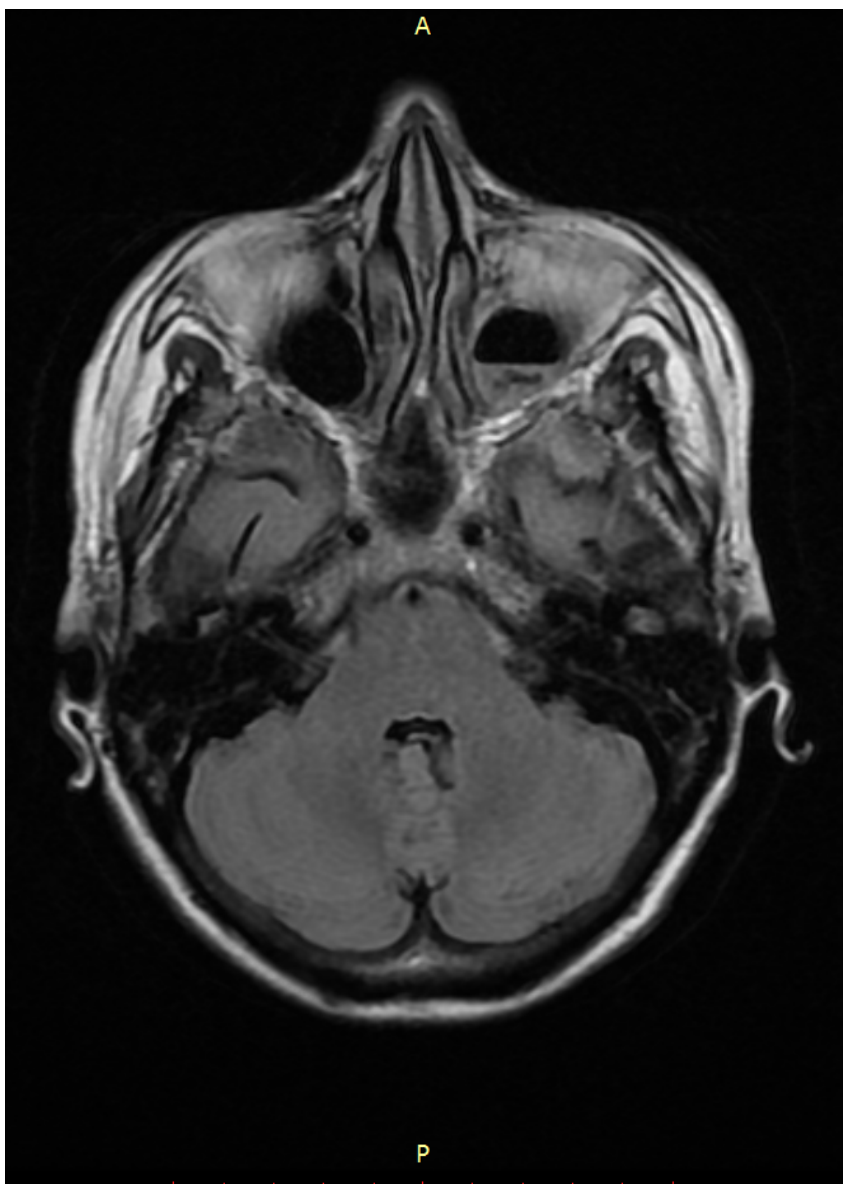

Figure 2. Axial FLAIR image 30 days after metronidazole interruption demonstrates complete reversal of signal changes

'Universidade Federal do Paraná, Hospital de Clínicas, Departamento de Neurologia, Curitiba PR, Brazil. Paula Teixeira MARQUES (ID https://orcid.org/0000-0003-1303-9273; Marcos Cristhiano LANGE (iD) https://orcid.org/0000-0002-0405-7157

Correspondence: Marcos Christiano Lange; Departamento de Neurologia HC/UFPR; Rua General Carneiro, 181 / $4^{\circ}$ andar; $80060-900$ Curitiba PR, Brasil; E-mail:langeneuro@gmail.com

Conflict of interest: There is no conflict of interest to declare.

Received on September 15, 2019; Received in its final form on October 21, 2019; Accepted on November 10, 2019. 


\section{References}

1. Goolsby TA, Jakeman B, Gaynes RP. Clinical relevance of metronidazole and peripheral neuropathy: a systematic review of the literature. Int J Antimicrob Agents. 2018 Mar;51(3):319-25. https:// doi.org/10.1016/j.ijantimicag.2017.08.033

2. Agarwal A, Kanekar S, Sabat S, Thamburaj K. Metronidazole-induced cerebellar toxicity. Neurol Int. 2016 Apr;8(1):6365. https://doi. org/10.4081/ni.2016.6365
3. Iemura T, Kitano T, Ishii A, Hishizawa M, Kondo T, Yamashita K. Metronidazole-induced encephalopathy during treatment for refractory diarrhea after cord blood transplantation. Clin J Gastroenterol. 2019 Oct;12(5):414-9. https://doi.org/10.1007/s12328-019-00959-x

4. Woodruff BK, Wijdicks EFM, Marshall WF. Reversible metronidazoleinduced lesions od the cerebellar dentate nuclei. N Engl J Med. 2002 Jan;346(1):68-9. https://doi.org/10.1056/NEJM200201033460117 